\title{
Emerging roles of ADAM and ADAMTS metalloproteinases in cancer
}

\author{
N. Rocks, G. Paulissen, M. El Hour, F. Quesada, C. Crahay, M. Gueders, J.M. Foidart, A. Noel, D. Cataldo \\ Department of Biology of Tumours and Development, GIGA-Research (Groupe Interdisciplinaire de Génoprotéomique Appliquée) and \\ Center for Experimental Cancer Research (CECR), University of Liège and CHU of Liège, Liège, Belgium
}

\begin{abstract}
A disintegrin and metalloproteinases (ADAMs) are a recently discovered family of proteins that share the metalloproteinase domain with matrix metalloproteinases (MMPs). Among this family, structural features distinguish the membrane-anchored ADAMs and the secreted ADAMs with thrombospondin motifs referred to as ADAMTSs. By acting on a large panel of membrane-associated and extracellular substrates, they control several cell functions such as adhesion, fusion, migration and proliferation. The current review addresses the contribution of these proteinases in the positive and negative regulation of cancer progression as mainly mediated by the regulation of growth factor activities and integrin functions.
\end{abstract}

Keywords: ADAM and ADAMTS proteins; Cancer; Cell proliferation; Apoptosis; Growth factors; Degradome

\section{INTRODUCTION}

Key features of malignant tumours are their abilities to invade surrounding tissues, to have access to the vascular and lymphatic systems, and to disseminate to distant organs by metastatic spreading. Cancer remains the second leading cause of death in Europe and the United States [1-3]. Accumulating evidence demonstrates the crucial role of proteolytic enzymes such as matrix metalloproteinases (MMPs) and closely related ADAMs (a disintegrin and metalloproteinase) and ADAMTSs (a disintegrin and metalloproteinase with thrombospondin motifs) in cancer development and progression. Although information about functions of ADAMs and ADAMTSs in cancers is still limited, recent studies have provided evidence of dysregulation of various ADAMs and ADAMTSs in different types of cancers. Therefore, these proteins have attracted attention of many research groups and functional analysis of ADAMs and ADAMTSs are ongoing based on the recent generation of mice deficient for some of these proteins. This review intends to discuss diverse functions of metalloproteinases implicated in cancer progression. Due to space constraints, we have chosen to concentrate our efforts on ADAMs and ADAMTSs, since MMPs have been extensively described in previous reviews [4-9]. Here, following a brief description of ADAMs and ADAMTSs, we explore their contribution to different steps of cancer progression.

\section{STRUCTURAL FEATURES OF ADAMS AND ADAMTSS}

The ADAM family members belong to the superfamily of zinc-dependent metalloproteinases also known as metzincins [10] and display sequence similarities with the reprolysin family of snake venomases. Two groups are distinguished in the adamalysin family: the membrane-anchored ADAMs and the secreted ADAMTSs.

The different domains composing ADAMs have independent but complementary functions endowing these proteins with features of proteinases [11] and adhesion molecules [12] (Fig. 1). The prodomain maintains the metalloproteinase domain inactive and has the ability to unveil catalytic site through a cystein switch mechanism upon activation by various processes. The furin-recognition site (RXXR sequence), located between pro and metalloproteinase domain, is believed to participate in intracellular activation of many ADAMs (ADAM-9, 12 , 15,17 ) by the action of furin-like proprotein convertases in the trans-Golgi network [13]. The metalloproteinase domain is characterized by a conserved HEXGH sequence shared with MMPs and confers the catalytic activity. It is worth noting that some ADAMs display alterations in this sequence resulting in a loss of proteolytic activity [14]. Although, the disintegrin domain has been widely described as being able to interact with integrin molecules and therefore mediating cell-cell and cell-matrix interactions $[12,15,16]$, caution should be advised regarding this feature. In a recent study by Takeda et al, the disintegrin domain has been shown not to be available for protein binding due to protein folding [17]. The disintegrin domain might thus be considered as a structural feature rather than an integrin ligand. The carboxy-terminal end is composed of a cystein-rich domain involved in cell-cell fusion [18], an EGF-like domain, a transmembrane domain and a cytoplasmic tail 
containing phosphorylation sites and SH3 binding domains [19]. At least 40 ADAMs have been described, 25 of which are expressed in Homo Sapiens. Among those, 19 display a proteolytic activity [20] (Table 1).

The complete human ADAMTS family comprises 19 ADAMTSs genes (Table 2) [21-23]. ADAMTSs are characterized by the presence of additional thrombospondin type I (TSP-I) motifs in their C-terminal part, while EGF-like, transmembrane and cytoplasmic domains are missing [24,25]. Some of them have one or two additional specific C-terminal modules such as a mucin domain (ADAMTS-7, and -12), a GON domain (ADAMTS-20 and -9), two CUB domains (ADAMTS-13) and/or a PLAC domain (ADAMTS-2, -3, -10, -12, $-14,-17,-19)$. Although ADAMTSs are soluble proteins, many of them appear to bind the extracellular matrix through their thrombospondin motifs or their spacer region [23]. With the exception of ADAMTS-10 and -12, ADAMTSs are regulated through a proteolytic processing occurring at the furin-like recognition site located between the pro- and catalytic domains [22,23].

The Tissue Inhibitor of Metalloproteinases (TIMPs) demonstrate selectivity in their inhibition of ADAMs and ADAMTSs which contrasts with their MMP-inhibitory features [26-29]. For example, ADAM-17 is exclusively inhibited by TIMP-3, ADAM-10 is sensitive to TIMP-1 and TIMP-3, but not to TIMP-2 and TIMP-4. The activity of ADAM-8 and -9 is not controlled by any TIMP [30]. TIMP-3 has the ability to inhibit ag-grecanase activity by targeting ADAMTS-4 while TIMP-1 and TIMP-2 display such an effect at higher range of concentrations [31].

Fig. 1. Structure of ADAM and ADAMTS proteinases. ADAM members are composed of common domains including propeptide (Pro), metalloproteinase (Metallo), disintegrin (Dis) with a conserved RGD domain for ADAM-15, cystein-rich (Cysrich), EGF-like (EGF), transmembrane (TM) and cytoplasmic domains. ADAMTS contain thrombospondin motifs (TSP1) and spacer domain (SP), but lack EGF-like, transmembrane and cytoplasmic domains. Some proteinases contain in addition a sequence recognized by furin-like enzymes (Fu) (see text).

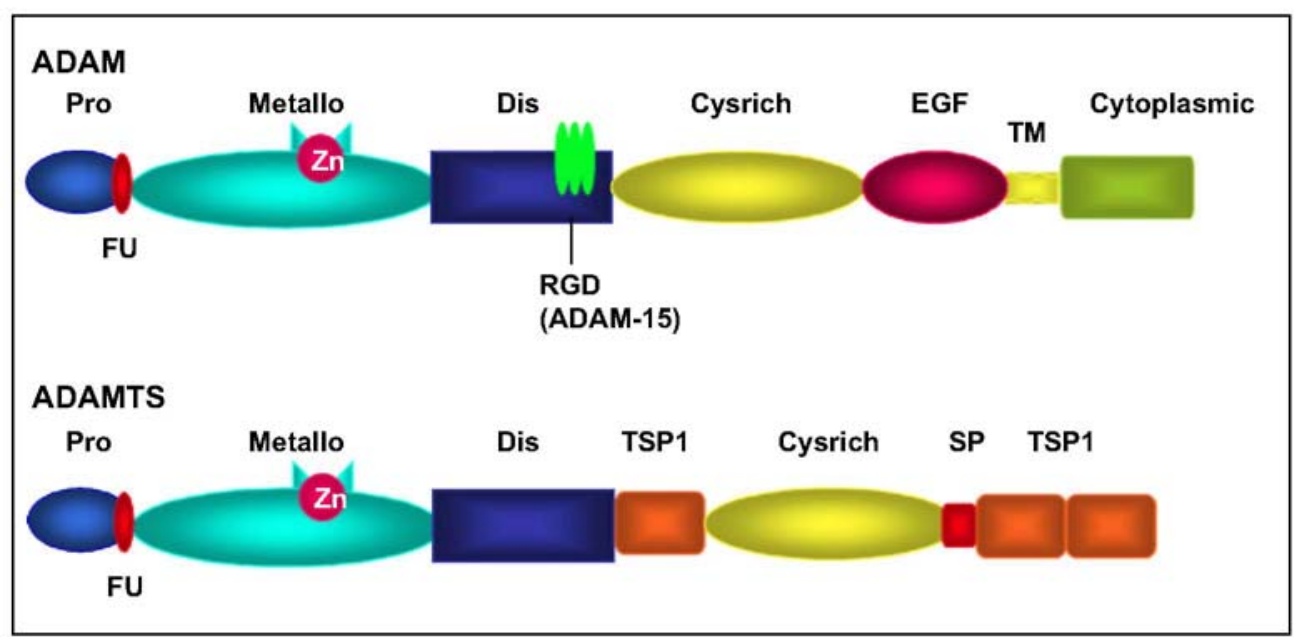

\section{IMPLICATION OF ADAMS AND ADAMTSS IN PHYSIOLOGY AND PATHOLOGY}

The complex but conserved structure of ADAM family members endows these proteins with various abilities leading to their key contribution to various physiological functions such as, for instance, fertilization $[15,32]$, neurogenesis [33], adipogenesis [34,35] and myogenesis [36]. ADAMs and ADAMTSs are involved in a complex molecular network of reciprocal interactions. Hence they can modulate cell responses to various signals by acting as cell surface sheddases. Of particular relevance is the activity of ADAM-17 (TACE) as a sheddase of membrane-bound pro-TNF- $\alpha$ [37,38] and of EGF receptor ligands leading to the release of active ligands [39]. Another illustrative example is the activation of NOTCH signalling by Notch ligand Delta shedding from the cell surface by ADAM-10 [40-42]. 
Table 1 : List of ADAMs with or without proteolytic activity

\begin{tabular}{|c|c|c|}
\hline ADAMs & Other names & Proteolytic activity (human) \\
\hline ADAM-1 a, b & $\begin{array}{l}\text { PH-30 alpha; } \\
\text { Fertilin alpha }\end{array}$ & - \\
\hline ADAM-2 & $\begin{array}{l}\text { PH- } 30 \text { beta; } \\
\text { Fertilin beta }\end{array}$ & - \\
\hline ADAM-3 & Cyritestin; tMDC I & - \\
\hline ADAM-4 & TMDCV & \\
\hline ADAM-5 & tMDC II & \\
\hline ADAM-6 & tMDC IV & - \\
\hline ADAM-7 & EAPI & - \\
\hline ADAM-8 & MS2, CD156 & + \\
\hline ADAM-9 & MDC9, meltrin gamma & + \\
\hline ADAM-10 & MADM; kuzbanian & + \\
\hline ADAM-11 & $\mathrm{MDC}$ & - \\
\hline ADAM-12 & Meltrin alpha & + \\
\hline ADAM-13 & (Xenopus laevis) & \\
\hline ADAM-14 & $\begin{array}{l}\text { adm-1, UNC-71 } \\
\text { (Caenorhabditis elegans) }\end{array}$ & \\
\hline ADAM-15 & Metargidin; MDC 15 & + \\
\hline ADAM-16 & MDC 16 (Xenopus laevis) & \\
\hline ADAM-17 & TACE & + \\
\hline ADAM-18 & TMDCIII & - \\
\hline ADAM-19 & Meltrin beta & + \\
\hline ADAM-20 & & + \\
\hline ADAM-21 & ADAM-31 & + \\
\hline ADAM-22 & MDC 2 & - \\
\hline ADAM-23 & MDC 3 & - \\
\hline ADAM-24 & Testase-1 & \\
\hline ADAM-25 & Testase-2 & \\
\hline ADAM-26 & Testase-3 & \\
\hline ADAM-27 & ADAM-18 & - \\
\hline ADAM-28 & $\begin{array}{l}\text { eMDCII, MDC-Lm, } \\
\text { MDC-Ls, TECADAM }\end{array}$ & + \\
\hline ADAM-29 & & - \\
\hline ADAM-30 & & + \\
\hline ADAM-31 & ADAM-21 & - \\
\hline ADAM-32 & & - \\
\hline ADAM-33 & & + \\
\hline ADAM-34 & Testase 4 & \\
\hline ADAM-35 & $\begin{array}{l}\text { Meltrin epsilon } \\
\text { (chicken, Gallus gallus) }\end{array}$ & \\
\hline ADAM-36 & Testase 6 & \\
\hline ADAM-37 & Testase 7 & \\
\hline ADAM-38 & Testase 8 & \\
\hline ADAM-39 & Testase 9 & \\
\hline ADAM-40 & Testase 10 & \\
\hline
\end{tabular}

Proteinase activity is shown only for human proteinases. These proteinases are not exclusively expressed in humans. In bold are ADAMs expressed in mouse but not in human. 
Table 2 : List of human ADAMTSs

\begin{tabular}{|c|c|c|}
\hline ADAMTS & Other names & Proteolytic activity \\
\hline ADAMTS-1 & C3-C5, METH1, KIAA1346 & + \\
\hline ADAMTS-2 & Procollagen N-proteinase & + \\
\hline ADAMTS-3 & KIAA0366 & + \\
\hline ADAMTS-4 & KIAA0688, aggrecanase-1, ADMP-1 & + \\
\hline ADAMTS-5 & ADAMTS-11, aggrecanase-2, ADMP-2 & + \\
\hline \multicolumn{3}{|l|}{ ADAMTS-6 } \\
\hline \multicolumn{3}{|l|}{ ADAMTS-7 } \\
\hline ADAMTS-8 & METH2 & + \\
\hline ADAMTS-9 & KIAA1312 & + \\
\hline \multicolumn{3}{|l|}{ ADAMTS-10 } \\
\hline ADAMTS-12 & UNQ1918, PR04389, AI605170 & + \\
\hline ADAMTS-13 & vWFCP, C9orf8 & + \\
\hline ADAMTS-14 & & + \\
\hline ADAMTS-15 & & + \\
\hline ADAMTS-16 & & + \\
\hline ADAMTS-17 & FLJ32769, LOC123271 & \\
\hline ADAMTS-18 & ADAMTS-21 & \\
\hline ADAMTS-19 & & \\
\hline ADAMTS-20 & & \\
\hline
\end{tabular}

ADAM and ADAMTS molecules have also been implicated in several pathologies [16,43-45]. ADAMTS-13 deficiency is responsible for thrombotic thrombocytopenic purpura characterized by the formation of microvascular von Willebrand Factor (vWF) and platelet-rich thrombi, associated with anaemia, renal failure and neurological dysfunction [46]. ADAM-17 expression and activity are increased in inflammatory bowel diseases [47]. A strong association has been established between ADAM-33 and asthma-related bronchial hyperresponsiveness in humans [48-50]. ADAM-8 expression is increased in an animal model of asthma following allergen exposure [51] and in the bronchi of human asthmatics [48,52]. ADAMTS-4 and TS-5 are involved in the turnover of aggrecan from cartilage resulting in loss of functionality of tissue and joint disability [53-56]. Studies have indicated that ADAMTS-5 is likely the major aggrecanase in cartilage metabolism and pathology [56]. Furthermore, its aggrecanase activity is 1000-fold greater than that of ADAMTS-4 under physiological conditions [57]. ADAM- 9 and -15 are upregulated in atherosclerosis along with integrins $\alpha 5 \beta 1$ and $\alpha v \beta 3[58]$.

The architecture of ADAMs and ADAMTSs, with domains that confer proteolytic activities and the ability to bind to diverse cell and extracellular matrix (ECM)-associated molecules, suggests that these enzymes may be functionally relevant to steps involved in cancer development and in metastatic dissemination of tumour cells.

The active metalloproteinase domain might indeed be needed to degrade extracellular matrix components and to shed growth factors and cytokines $[8,59]$, contributing in this way to the control of cell proliferation, migration and angiogen-esis [60]. Adhesion and migration of cells might be regulated by the disintegrin or cystein-rich domains whose importance has been evidenced by different studies [12,61-63].

These data illustrate how much ADAMs and ADAMTSs are multifunctional proteins and suggest that they may serve as regulators of proteolytic and non-proteolytic events occurring during cancer progression. To date, only few data are available about the roles of those proteins in cancer initiation and progression. Dysregulation of ADAM and ADAMTS expression has been reported in different types of cancer by RT-PCR profiling and microarray analysis [64-67]. The picture is rendered complex by the existence of different isoforms resulting from alternative splicing described in ADAM-8, -9, -10, -11, -12, -15, -19, -22, -28, -29, -30 and -33 or ADAMTS-4 and TS-6 genes [36,50,51,68-77] or from putative post-translational modulations [23] resulting from a processing of the molecule by the metalloproteinase domain itself [78] or by other MMPs [79].

\section{RELEVANCE OF ADAMS AND ADAMTSS IN DIFFERENT STEPS OF CANCER PROGRESSION}

Here, we review different studies suggesting a predominant role for ADAMs and ADAMTSs in processes related to cancer progression such as the regulation of cell cycle and angiogenesis. 


\subsection{ADAMs and ADAMTSs in cell proliferation and apoptosis}

Several proteolytically active ADAMs and ADAMTSs regulate cell proliferation by cleaving growth factors or cell surface proteins. Ligands for several growth factor receptors are processed by ADAM family members. Among them, EGF receptor ligands (heparin-binding EGF (HB-EGF), amphiregulin, betacellulin, epiregulin) are synthesized as transmembrane precursors and require ectodomain shedding for activation [59,80]. ADAM-17 has been shown to play a key role in such a process [60,81]. Amphiregulin released by ADAM-17 cleavage enhances cell proliferation of cancer cells $[82,83]$. HB-EGF is a potent inducer of tumour growth and angiogenesis [84]. Shedding of EGFR-ligands by ADAM-17 is increased upon cell stimulation by phorbol esters [85] and ADAM-17 also contributes to the release of bioactive epigen, a highly mitogenic ligand of EGFR which has been implicated in cancer [86]. Reciprocally, a long term treatment of different cell types with EGF leads to a marked enhancement of ADAM-17 by increasing its half-life and promotes thereby the shedding of different substrates [87].

ADAM-10 contributes to E-cadherin shedding [88,89]. The subsequent release of soluble E-cadherin in the extracellular milieu leads to the abrogation of cell-cell contacts, thereby facilitating cell migration. ADAM-10 also contributes to cell proliferation by modulating $\beta$-catenin signalling through E-cadherin shedding and increasing gene cyclin D1 levels [90]. Such processes should be of particular importance in embryonic development since ADAM-10 knock-out (KO) embryos suffer from cell growth arrest and apoptosis associated with an overexpression of full-length E-cadherin [89].

Moreover, ADAM proteinases control cell apoptosis. Indeed, in a mammary cancer model induced by the expression of polyoma middle T oncoprotein, ADAM-12 has been shown to increase stromal cell apoptosis and decrease tumour cell apoptosis [91]. INCB3619, a selective inhibitor of a subset of ADAM proteinases, blocks the shedding of ErbB ligands, reduces ErbB ligand shedding in vivo and inhibits ErbB pathway signalling, tumour cell proliferation and survival [92]. Altogether, these data emphasize the key role of ADAM proteinases in the regulation of cell proliferation and apoptosis although the dissection of precise mechanisms will necessitate further investigations.

\subsection{Roles of ADAMs and ADAMTSs in angiogenesis}

Angiogenesis consists in the formation of new blood vessels devoted to vascularise the tumour tissue and is considered as a crucial event in solid tumour growth and progression. Angiogenesis process is under the dependence of a balance of pro- and antiangiogenic factors [93-95]. Proteinases have been initially considered as positive regulators of angiogenesis but recent studies have evidenced complex and sometimes opposite roles of MMPs, ADAMs and ADAMTSs in regulating tumoral angiogenesis [5,83,95-98]. Interestingly, ADAMTS-1 and ADAMTS-8 have been proven to be antiangiogenic factors [99]. This anti-angiogenic effect is thought to be mediated by their thrombospondin motifs through their interaction with CD36, a membrane glycoprotein receptor of endothelial cells [100,101] or directly through VEGF binding [102]. Multiple mechanisms have been proposed to explain the inhibition of angiogenesis by members of the ADAM and ADAMTS family. Among those, it is worth noting that ADAMTS-1 comprises TSP-1 repeats which may contribute to the antiangiogenic activity by trapping vascular endothelial growth factor $(\mathrm{VEGF})_{165}[100,102]$. Taking these data together, ADAMTS-1 C-terminal domain should be considered as an anti-tumour and anti-metastatic region [78,103]. The ADAMTS-1 story will probably mature in the next few years since some authors have also shown that overexpression of full-length ADAMTS-1 in CHO cells enhances tumour growth [103] and promotes pulmonary metastasis of TA3 mammary carcinoma or Lewis lung cells [78]. One possible approach to rationalize the apparently contradictory information on ADAMTS-1 is to consider that this molecule undergoes auto-proteolytic cleavage that can account for pro- or anti-metastatic effects depending on the cleavage site [78]. Indeed, these dual pro and anti-tumoral activities can be explained by a proteolytic cleavage of the substrate-binding site impairing the binding of the catalytic site to amphiregulin or HB-EGF [78]. This cleavage probably also unveils TSP-1 motifs' antitumour activity. Therefore, C-terminal processing of ADAMTS-1 affects protein bioactivity and may account for some apparently controversial effects.

ADAM-15 has also been found to bear angiogenesis regulatory properties. ADAM-15 is expressed by smooth muscle cells, umbilical vein endothelial cells and more preferentially by activated endothelial cells [104]. The recombinant disintegrin domain (RDD) of ADAM-15 has been reported as a potent inhibitor of angiogenesis [105]. In vivo, ADAM-15 RDD induces a reduction of MDA-MB-231 tumour growth associated with less tumour vascularization. Transgenic B16F10 melanoma cells form less metastasis in mouse lungs after turning on RDD expression [105]. Angiogenesis is inhibited in ADAM-15-deficient mice in a model of retinopathy [106]. Mechanisms implicating ADAM-15 in the regulation of angiogenesis could be related to the presence of 
Arg-Gly-Asp (RGD) sequence in the disintegrin domain which binds integrins. Indeed, ADAM-15 decreases integrin $\alpha v \beta_{3}$ /vitronectin-mediated ovarian cancer cell adhesion and motility in a RGD-dependent manner [107]. However, although tumours developed from melanoma cells implanted subcutaneously are smaller in ADAM$15^{-/-}$mice, no difference in tumour vascularity has been observed between wild type and mutant mice [106]. The exact role of ADAM-15 during angiogenesis appears complex and requires further investigations.

\section{CONTRIBUTION OF ADAMS AND ADAMTSS IN DIFFERENT TYPES OF CANCER}

\subsection{Lung cancer}

In 2006 , lung cancer accounted for about $13 \%$ of all cancer diagnoses. Smoking is by far the most important risk factor for lung cancer and about $87 \%$ of lung cancers are thought to result directly from smoking. Tobacco smoke contains numerous carcinogens and primary bronchial epithelial cells as well as bronchial cell lines exposed to smoke components show an increased proliferation rate associated with EGFR phosphorylation [108]. This could be at least in part mediated by ADAM-17 which can activate several EGFR ligands [108]. ADAM-17 is indeed upre-gulated in non-small cell lung carcinoma (NSCLC) and is required for heregulin3 (HER3) signalling but also for EGFR-li-gand-dependent signalling and inhibition of ADAMs affects the activation of many ErbB ligands and, as a matter of consequence, multiple ErbB pathways in NSCLC [109].

Dysregulation of the production of several ADAMs has been documented in lung cancers. As demonstrated by tissue microarray analysis, a strong ADAM-8 expression is present in NSCLC and correlates with clinical stage of the disease [65]. As induction of ADAM-8 production increases the invasive phenotype of cancer cells, this molecule might play a role in promoting disease dissemination. ADAM-9 mRNA and protein expression levels are enhanced in EBC-1 lung cancer cell line displaying a tropism for brain metastasis as compared to parent EBC-1 or EBC-1 cell line with a tropism for bone tissue [110]. Overexpression of ADAM-9 in EBC-1 and A549 lung cancer cells results in an increase of NGF-induced invasion and a higher adhesion of cells to brain tissue. A549 cells over-expressing ADAM-9 have the potential to develop brain metastasis when injected intravenously.

We recently reported an increase in ADAM-12 mRNA and protein levels while ADAMTS-1 levels are decreased in NSCLC when compared to non-cancerous tissues [111]. Whereas ADAMTS-1 is mainly produced by normal bronchial epithelium, ADAM-12 is expressed in vast majority by cancer cells as demonstrated by immunohistochemistry, suggesting that ADAM-12 plays a role in the cascade of events leading to invasive capacities. ADAM-12 might be an important mediator of biological processes leading to tumour-related angiogenesis and thereby tumour development. ADAM-28, which cleaves insulin-like growth factor binding protein-3 (IGFBP-3) [112], is found to be about 16-fold over-expressed in NSCLC. This proteinase is mainly present in carcinoma cells and correlates with cancer cell proliferation and lymph node metastasis [113]. ADAM-15, $\alpha$ v and $\beta 3$ integrins are expressed in small cell lung carcinoma (SCLC) and NSCLC cell lines [114] and ADAM-15 expression is higher in tumoral cells than in normal epithelial cells of pulmonary tumours.

Interestingly, ADAMTS-8, a potent anti-angiogenic ADAMTS is downregulated in most primary NSCLC [115] due to abnormal promoter hypermethylation [115].

\subsection{Brain tumours}

Both ADAM-22 and ADAM-23, displaying high sequence similarities with ADAM-11, are restricted to the brain. They are implicated in cell-cell and cell-matrix interactions through their binding to integrins and extracellular matrix and, consequently, might be involved in neural development [75]. There is evidence for a modulation of the expression of ADAM and ADAMTS genes in brain tumours since cytoplasmic variants of ADAM-22 are differently expressed in normal human brain tissue and gliomas [116]. Moreover, ADAM-22 inhibits astrocyte proliferation by interaction of its disintegrin domain with cell surface integrins [117]. Brain tumours, often invading surrounding parenchyma, could thus display a modulated proteinase expression facilitating tumour cell infiltration and/ or angiogenesis. Some ADAMTS proteinases (ADAMTS-8 and ADAMTS-13) display lower levels of expression in brain tumours as compared to normal brain tissue [118,119]. In sharp contrast, ADAMTS-4 and TS-5 are overexpressed in human glioblastomas and could be responsible for brevican cleavage and contribute to invasiveness of glioblastoma cells [120].

A contribution of ADAM-17 to glioma cell invasiveness through activation of the EGFR signal pathway under hypoxic conditions has been suggested. Indeed, brain tumour cell lines cultured under hypoxic conditions demonstrated an upregula-tion of ADAM-17 expression levels, whose activity correlated with increased tumour cell invasion [121]. 
Similarly, ADAM-8 and ADAM-19 mRNA are upregulated in primary brain tumours and their expression and activity are correlated with invasiveness of glioma cells [122]. The membrane-bound ADAM-12 variant is overexpressed in glioblastomas [123] and a treatment of cultured glioblastoma cells with an ADAM-12 inhibitor decreases the production of mature HB-EGF indicating that ADAM-12-HB-EGF pathway might be of biological significance in those cells.

\subsection{Prostate cancer}

Development of prostate cancer is androgen-dependent in early stages but cell growth can become androgenindependent $[124,125]$. This androgen-dependency could interfere with ADAM-related regulation processes since the mRNA expression of several ADAMs is regulated by androgens [126]. Indeed, ADAM-9, -10, -11, -15 and -17 are expressed in prostate cancer cells and treatment of androgen-dependent cancer cells with dihydrotestosterone leads to an upregulation of ADAM-9 and -10 mRNAs while ADAM-17 mRNA is downregulated. ADAM-9 protein levels are elevated in malignant as compared with benign prostate tissues. Androgen or serum starvation enhances ADAM-9 protein expression in androgen-receptor-positive prostate cancer cells [127]. ADAM-8 protein expression has been demonstrated to be significantly associated with higher cancer stages including positive nodal status, and higher Gleason scores [128].

ADAMTS-13 activity is mildly diminished in prostate tumours even if these rates are not related to metastasis and low ADAMTS-13 activity could diminish vWF (Von Willebrand Factor) cleavage resulting in an accumulation of highly polymeric vWF, facilitating adhesive interactions between circulating tumour cells and platelets. Moreover, other authors have reported thatpatients with advanced stage and metastasis of cancer display lower ADAMTS-13 levels [129,130]. Prostate stroma cells constitutively express ADAMTS-1, -4, -5, -9, -15 as well as TIMP-3 in contrast to some prostate cancer cell lines [131].

ADAM-15 is overexpressed in aggressive prostate adenocarcinoma, correlates with cancer stages, and might be a marker for a more aggressive prostate cancer subtype [132]. ADAM-10 is specifically expressed on the cell surface of normal epithelial cells [133] while tumour cells show mainly nuclear staining for ADAM-10 suggesting an interaction with nuclear proteins or DNA [134].

\subsection{Liver carcinoma}

Upon the occurrence of liver injury, activated hepatic stellate cells (HSC) contribute to the inflammatory response by secretion of MMPs resulting in ECM remodelling and increased matrix deposition [135]. ADAMs are also implicated in hepatocellular carcinoma development [136]. Indeed, ADAM-17 contributes to EGFRligand release and induction of cell proliferation and invasion [137]. A link has been established between TGF$\beta 1$ and cell proliferation since TGF- $\beta 1$ induces a rapid activation of ADAM-17 leading in turn to EGFR signalling [138]. In activated hepatic stellate cells, TGF- $\beta 1$ induces ADAM-12 expression which might also participate in tumour progression [139]. It is worth pointing out that a recent study identified ADAM-12 as a partner of TGF $\beta$ receptor II signalling where it stabilizes the TGF $\beta$ RII protein and potentiates Smad-mediated signalling [140]. Hence, ADAM-12 might contribute to growth inhibitory signalling in normal epithelial cells which is lost during tumour progression.

ADAM-9 promotes invasiveness of liver metastatic carcinoma cells by degrading basement membrane components such as laminin-1 [141]. ADAM-9 is differentially expressed in stromal or epithelial cells and a soluble variant of ADAM-9 is secreted by activated hepatic stellate cells but not carcinoma cells or hepatocytes, indicating that stroma production of ADAM-9 might be of particular importance.

ADAM-17 mRNA levels are higher in hepatocellular carcinomas than in paired non-cancerous liver tissues suggesting that this proteinase might be implicated in tumour invasiveness by either activating EGFR by amphiregulin [108] or TGF-alpha [142].

\subsection{Breast cancer}

Several ADAMs and ADAMTSs are found to be modulated in breast cancer patients $[66,132,143]$. ADAM-9, 15, -17 mRNA levels are higher in breast cancer surgical samples whereas ADAM-10 mRNA levels are not modulated [132, 143]. ADAM-12, as previously stated in the "proliferation and apoptosis" section of this review, is an apoptosis-modulating gene which is upregulated in human breast cancer tissues whereas nonmalignant breast lesions express very low amounts of the proteinase. Accordingly, the overexpression of soluble ADAM-12 lacking the cytoplasmic tail (secreted splice variant of ADAM-12) accelerates the development of 
tumour by delaying tumour cell apoptosis [91]. It is worth noting that urine ADAM-12 might be a potentially important non-invasive biomarker in breast cancer since levels are enhanced in breast cancer patients [143].

In vitro studies have shown that overexpression of ADAM-17 in breast cancer cells increases invasion and proliferation [144]. Inversely, targeting this proteinase reverts the malignant phenotype in breast cancer cells by preventing shedding of TGF- $\alpha$ and amphiregulin [145]

Proteinase activation appears to be by itself of particular importance in carcinogenesis suggesting an involvement of metalloproteinase activity in cancer development. Indeed, ADAM-17 ratio of active/pro protein levels increased progressively from normal breast tissue, to primary breast cancer, and to lymph node metastases. In primary tumours, the active form of ADAM-17 correlates with levels of urokinase plasminogen activator and proliferating cell nuclear antigen [144].

Some precise mechanisms of action have been proposed for members of the ADAM family. For example, active ADAM-28 which is overexpressed in breast carcinoma cells contributes to the regulation of cell proliferation through IGFBP-3 cleavage, enhancing the bioavailability of IGF-I [146]. Alternative splicing could also be an important tool used by cancer cells to acquire an invasive phenotype since, for example, different isoforms of ADAM-9 proteins and ADAM-15 mRNA have been detected in breast cancer cells [74, 147]. This last finding could give the opportunity to set up a powerful diagnostic tool by studying the differential production of ADAM9 or -15 domains. Some ADAMs could be relevant markers of therapeutic response. ADAM-9 and ADAM-11 mRNA levels in tumours are indeed associated with better response to tamoxifen therapy and ADAM-9 protein production is an indicator of poor prognosis [147,148]. Patients displaying elevated levels of ADAMTS-8 and low levels of ADAMTS-15 have a general poor clinical outcome [149].

\subsection{Gastric and colon carcinoma}

ADAM-10, which is found to be overexpressed in vitro after gastric cell infection, could establish a link between Helicobacter pylori-induced inflammation and carcinogenesis in stomach. In vivo, ADAM-10 and -17 are overexpressed in antral mucosa during $H$. pylori infection and ADAM-9, -10, -12, -15, and -17 are increased in gastric tumours $[150,151]$. ADAM-10 acts through EGFR ligand shedding leading to gastric cell proliferation [151-153]. In colon carcinomas, ADAM-17 is overexpressed independently of tumour stage or grade and is involved in tumour growth and angiogenesis possibly via an autocrine/paracrine pathway implicating EGFR [154].

In vitro expression of the alternative spliced secreted variant ADAM-9S (short) in a non-invasive colon cell line induces a highly invasive phenotype. ADAM-9 is overexpressed in a colon cell line and is co-localized with Ecadherin suggesting a potential role in E-Cadherin-mediated metastasis $[141,155]$. ADAMTS-1 has recently been recognized as a novel gene inactivated through promoter hypermethylation in colorectal tumour development [156].

\subsection{Kidney, bladder carcinoma}

EGFR signalling appears important in the development of kidney cancer since inhibition of ADAM-17 by a dominant negative ADAM-17 mutant prevents pro-HB-EGF cleavage, EGFR activation and cell proliferation in kidney carcinoma cells [81]. As previously described in other types of cancers, ADAM-12 mRNA was found to be overexpressed in bladder cancer and ADAM-12 levels correlated with disease stage. ADAM-12 could also be an interesting biomarker since it is present in higher levels in the urine from patients with bladder cancer [64].

\subsection{Pancreatic carcinoma}

ADAM-9, -10 and -17 are expressed in pancreatic tissues but are restricted to specific compartments. Analysis of mRNA expression levels in microdissected cancer samples shows an overexpression of ADAM-9 and -15 proteinases in pancreatic tumour cells [157]. In contrast, ADAMTS-1 expression is lower in pancreatic tumours and patients displaying higher levels of ADAMTS-1 are subject to more retroperitoneal invasion and lymph node metastasis associated with poor survival [158]. ADAM-17, only weakly expressed in normal pancreatic tissues, is overexpressed in all pancreatic ductal adenocarcinoma (PDAC) and pancreatic cancer cell lines. The role of ADAM-17 in pancreatic cancer is underscored by experiments showing that inhibition of ADAM-17 gene expression, by using small interfering RNA (siRNA) technique, affects invasiveness of tumour cells [159]. 


\section{CONCLUSION}

ADAMs and ADAMTSs are proteins displaying some structural features conferring the potency to display multiple functions. ADAMs and ADAMTSs play crucial roles in biological processes as various as cellular adhesion, cell fusion, shedding of plasma membrane-associated proteins and intracellular signalling. In the present review, we have presented recent data showing that altered expression of ADAMs and ADAMTSs has been found in diverse tumour types which suggests that these proteins are involved in different steps of cancer progression including carcinogenesis (Fig. 2) by regulating tumour cell proliferation, apoptosis and invasiveness.

However, the exact role of these proteinases in the initiation or progression of the disease is generally still poorly understood. Of interest is that some of these proteinases might be useful tools as biomarkers for early cancer diagnostic.

Studies using broad-spectrum MMP inhibitors have provided numerous data in the literature. However, no synthetic inhibitor has shown promising results in clinical trials. This might be explained by the non-specificity of inhibitors used, which might also inhibit other proteinases such as ADAMs and ADAMTSs themselves embedded in complex networks of interactions regulating many biological processes including some protective mechanisms. Nevertheless, the design of specific agents is a real challenge since more than fifty similar proteinases exist in humans (23 MMPs, 13 ADAMs and 19 ADAMTSs). Some recent advances might offer in the next future the opportunity to design such specific inhibitors by using e.g. siRNAs or monoclonal antibodies.

The precise understanding of the exact role played by each ADAM and ADAMTS in cancer appears of particular importance in the perspective to design new therapeutic strategies based on the control or inhibition of those proteinases.

Fig. 2. Implication of ADAM molecules in different processes contributing to cancer development. ADAMs are composed of distinct domains endowing the proteins with multiple functions.

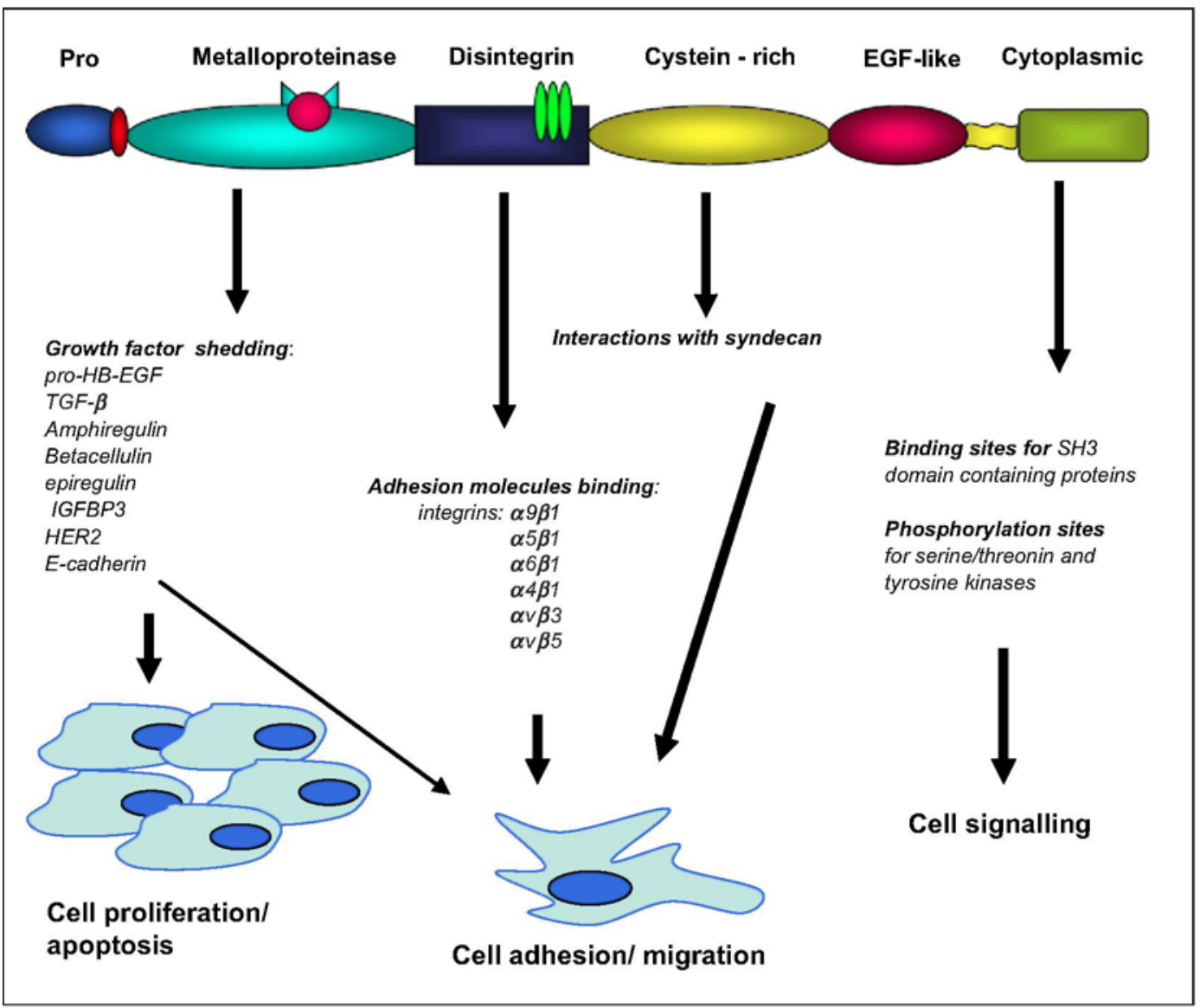


Published in: Biochimie (2008), vol. 90, iss. 2, pp. 369-379

Status: Postprint (Author's version)

\section{Acknowledgments}

This work was supported by grants from the Commission of European Communities (FP6), the Fonds National de la Recherche Scientifique (F.N.R.S., Belgium), the Fédération Belge Contre le Cancer, the Fonds spéciaux de la Recherche (University of Liège), the Centre Anticancéreux près l'Université de Liège, the D.G.T.R.E. from the «Région Wallonne», the F.S.E. (Fonds Social Européen).

\section{References}

[1] C.A. Butler, K.M. Darragh, G.P. Currie, W.J. Anderson, Variation in lung cancer survival rates between countries: do differences in data reporting contribute? Respir. Med 100 (2006) 1642-1646.

[2] K. Shibuya, M. Inoue, A.D. Lopez, Statistical modeling and projections of lung cancer mortality in 4 industrialized countries, Int. J. Cancer 117 (2005) 476-485.

[3] J. Ferlay, P. Autier, M. Boniol, M. Heanue, M. Colombet, P. Boyle, Estimates of the cancer incidence and mortality in Europe in 2006, Ann. Oncol 18 (2007) 581-592.

[4] K.J. Greenlee, Z. Werb, F. Kheradmand, Matrix metalloproteinases in lung: multiple, multifarious, and multifaceted, Physiol. Rev. 87 (2007) 69-98.

[5] M.M. Handsley, D.R. Edwards, Metalloproteinases and their inhibitors in tumor angiogenesis, Int. J. Cancer 115 (2005) $849-860$.

[6] A. Noel, M. Jost, E. Maquoi, Matrix metalloproteinases at cancer tumor-host interface. Semin. Cell Dev. Biol, in press, doi: 10.1016/j.semcdb.2007.05.011

[7] CM. Overall, R.A. Dean, Degradomics: systems biology of the protease web. Pleiotropic roles of MMPs in cancer, Cancer Metastasis Rev. 25 (2006) 69-75.

[8] CM. Overall, O. Kleifeld, Tumour microenvironment - opinion: validating matrix metalloproteinases as drug targets and anti-targets for cancer therapy, Nat. Rev. Cancer 6 (2006) 227-239.

[9] A. Page-McCaw, A.J. Ewald, Z. Werb, Matrix metalloproteinases and the regulation of tissue remodelling, Nat. Rev. Mol. Cell Biol. 8 (2007) 221-233

[10] T.G. Wolfsberg, P. Primakoff, D.G. Myles, J.M. White, ADAM, a novel family of membrane proteins containing a disintegrin and metallopro-tease domain: multipotential functions in cell-cell and cell-matrix interactions, J. Cell Biol. 131 (1995) $275-278$.

[11] C.P Blobel, Metalloprotease-disintegrins: links to cell adhesion and cleavage of TNF alpha and Notch, Cell 90 (1997) 589-592.

[12] X.P. Zhang, T. Kamata, K. Yokoyama, W. Puzon-McLaughlin, Y. Takada, Specific interaction of the recombinant disintegrin-like domain of MDC-15 (metargidin, ADAM-15) with integrin alphavbeta3, J. Biol. Chem. 273 (1998) 7345-7350.

[13] F. Loechel, B.J. Gilpin, E. Engvall, R. Albrechtsen, U.M. Wewer, Human ADAM 12 (meltrin alpha) is an active metalloprotease, J. Biol. Chem. 273 (1998) 16993-16997.

[14] R.A. Black, J.M. White, ADAMs: focus on the protease domain, Curr. Opin. Cell Biol. 10 (1998) 654-659.

[15] K. Eto, C. Huet, T. Tarui, et al., Functional classification of ADAMs based on a conserved motif for binding to integrin alpha 9beta 1 : implications for sperm-egg binding and other cell interactions, J. Biol. Chem. 277 (2002) 17804-17810.

[16] K. Reiss, A. Ludwig, P. Saftig, Breaking up the tie: disintegrin-like metalloproteinases as regulators of cell migration in inflammation and invasion, Pharmacol. Ther 111 (2006) 985-1006.

[17] S. Takeda, T. Igarashi, H. Mori, S. Araki, Crystal structures of VAP1 reveal ADAMs' MDC domain architecture and its unique Cshaped scaffold, EMBO J 25 (2006) 2388-2396

[18] A.P. Huovila, E.A. Almeida, J.M. White, ADAMs and cell fusion, Curr. Opin. Cell Biol. 8 (1996) 692-699.

[19] A.L. Stone, M. Kroeger, Q.X. Sang, Structure-function analysis of the ADAM family of disintegrin-like and metalloproteinasecontaining proteins (review), J. Protein Chem. 18 (1999) 447-465.

[20] D.F. Seals, S.A. Courtneidge, The ADAMs family of metalloproteases: multidomain proteins with multiple functions, Genes Dev. 17 (2003) 7-30. 
Published in: Biochimie (2008), vol. 90, iss. 2, pp. 369-379

Status: Postprint (Author's version)

[21] S.S. Apte, A disintegrin-like and metalloprotease (reprolysin type) with thrombospondin type 1 motifs: the ADAMTS family, Int. J. Biochem. Cell Biol. 36 (2004) 981-985.

[22] S. Cal, A.J. Obaya, M. Llamazares, C. Garabaya, V. Quesada, C. Lopez-Otin, Cloning, expression analysis, and structural characterization of seven novel human ADAMTSs, a family of metalloproteinases with disintegrin and thrombospondin-1 domains, Gene 283 (2002) 49-62.

[23] S. Porter, I.M. Clark, L. Kevorkian, D.R. Edwards, The ADAMTS metalloproteinases, Biochem. J. 386 (2005) 15-27.

[24] G.P. Kaushal, S.V. Shah, The new kids on the block: ADAMTSs, potentially multifunctional metalloproteinases of the ADAM family, J. Clin. Invest 105 (2000) 1335-1337.

[25] B.L. Tang, W. Hong, ADAMTS: a novel family of proteases with an ADAM protease domain and thrombospondin 1 repeats, FEBS Lett. 445 (1999) 223-225.

[26] A. Amour, C.G. Knight, A. Webster, et al., The in vitro activity of ADAM-10 is inhibited by TIMP-1 and TIMP-3, FEBS Lett. 473 (2000) 275-279.

[27] M. Kashiwagi, M. Tortorella, H. Nagase, K. Brew, TIMP-3 is a potent inhibitor of aggrecanase 1 (ADAM-TS4) and aggrecanase 2 (ADAMTS5), J. Biol. Chem. 276 (2001) 12501-12504.

[28] G. Murphy, V. Knauper, M.H. Lee, et al., Role of TIMPs (tissue inhibitors of metalloproteinases) in pericellular proteolysis: the specificity is in the detail, Biochem. Soc. Symp 70 (2003) 65-80.

[29] H. Nagase, K. Brew, Designing TIMP (tissue inhibitor of metalloproteinases) variants that are selective metalloproteinase inhibitors, Biochem. Soc. Symp 70 (2003) 201-212.

[30] A. Amour, C.G. Knight, W.R. English, et al., The enzymatic activity of ADAM8 and ADAM9 is not regulated by TIMPs, FEBS Lett 524 (2002) 154-158.

[31] G.J. Wayne, S.J. Deng, A. Amour, et al., TIMP-3 inhibition of ADAMTS-4 (Aggrecanase-1) is regulated by interactions between aggrecan and the C-terminal domain of ADAMTS-4, J. Biol. Chem. 282 (2007) 20991-20998.

[32] R. Yuan, P. Primakoff, D.G. Myles, A role for the disintegrin domain of cyritestin, a sperm surface protein belonging to the ADAM family, in mouse sperm-egg plasma membrane adhesion and fusion, J. Cell Biol. 137 (1997) 105-112.

[33] P. Yang, K.A. Baker, T. Hagg, The ADAMs family: coordinators of nervous system development, plasticity and repair, Prog. Neurobiol. 79 (2006) 73-94.

[34] N. Kawaguchi, X. Xu, R. Tajima, et al., ADAM 12 protease induces adi-pogenesis in transgenic mice, Am. J. Pathol 160 (2002) 18951903.

[35] M. Masaki, T. Kurisaki, K. Shirakawa, A. Sehara-Fujisawa, Role of meltrin \{alpha\} (ADAM12) in obesity induced by high- fat diet, Endocrinology 146 (2005) 1752-1763.

[36] B.J. Gilpin, F. Loechel, M.G. Mattei, E. Engvall, R. Albrechtsen, U.M. Wewer, A novel, secreted form of human ADAM 12 (meltrin alpha) provokes myogenesis in vivo, J. Biol. Chem. 273 (1998) 157-166.

[37] R.A. Black, C.T. Rauch, C.J. Kozlosky, et al., A metalloproteinase dis-integrin that releases tumour-necrosis factor-alpha from cells, Nature 385 (1997) 729-733.

[38] M.L. Moss, S.L. Jin, M.E. Milla, et al., Cloning of a disintegrin metalloproteinase that processes precursor tumour-necrosis factor-alpha, Nature 385 (1997) 733-736

[39] U. Sahin, G. Weskamp, K. Kelly, et al., Distinct roles for ADAM10 and ADAM17 in ectodomain shedding of six EGFR ligands, J. Cell Biol. 164 (2004) 769-779.

[40] D. Hartmann, B. de Strooper, L. Serneels, et al., The disintegrin/metallo-protease ADAM 10 is essential for notch signalling but not for alpha-secretase activity in fibroblasts, Hum. Mol. Genet. 11 (2002) 2615-2624.

[41] H. Qi, M.D. Rand, X. Wu, et al., Processing of the notch ligand delta by the metalloprotease Kuzbanian, Science 283 (1999) $91-94$.

[42] K.L. Taylor, A.M. Henderson, C.C. Hughes, Notch activation during endothelial cell network formation in vitro targets the basic HLH transcription factor HESR-1 and downregulates VEGFR-2/KDR expression, Microvasc. Res. 64 (2002) 372-383.

[43] G.C. Jones, ADAMTS proteinases: potential therapeutic targets? Curr. Pharm. Biotechnol 7 (2006) 25-31.

[44] C.B. Kern, W.O. Twal, C.H. Mjaatvedt, et al., Proteolytic cleavage of versican during cardiac cushion morphogenesis, Dev. Dyn 235 (2006) 2238-2247. 
Published in: Biochimie (2008), vol. 90, iss. 2, pp. 369-379

Status: Postprint (Author's version)

[45] A.M. Manso, L. Elsherif, S.M. Kang, R.S. Ross, Integrins, membrane-type matrix metalloproteinases and ADAMs: potential implications for cardiac remodeling, Cardiovasc. Res. 69 (2006) 574-584.

[46] G.G. Levy, W.C. Nichols, E.C. Lian, et al., Mutations in a member of the ADAMTS gene family cause thrombotic thrombocytopenic purpura, Nature 413 (2001) 488-494.

[47] J. Brynskov, P. Foegh, G. Pedersen, et al., Tumour necrosis factor alpha converting enzyme (TACE) activity in the colonic mucosa of patients with inflammatory bowel disease, Gut 51 (2002) 37-43.

[48] S.C. Foley, A.K. Mogas, R. Olivenstein, et al., Increased expression of ADAM33 and ADAM8 with disease progression in asthma, J. Allergy Clin. Immunol 119 (2007) 863-871.

[49] S.T Holgate, Y. Yang, H.M. Haitchi, et al., The genetics of asthma: ADAM33 as an example of a susceptibility gene, Proc. Am. Thorac. Soc. 3 (2006) 440-443.

[50] P. Van Eerdewegh, R.D. Little, J. Dupuis, et al., Association of the ADAM33 gene with asthma and bronchial hyperresponsiveness, Nature 418 (2002) 426-430.

[51] N.E. King, N. Zimmermann, S.M. Pope, et al., Expression and regulation of a disintegrin and metalloproteinase (ADAM) 8 in experimental asthma, Am. J. Respir. Cell Mol. Biol. 31 (2004) 257-265.

[52] G. Paulissen, N. Rocks, F. Quesada-Calvo, et al., Expression of ADAMs and their inhibitors in sputum from patients with asthma, Mol. Med 12 (2006) 171-179.

[53] C.J. East, H. Stanton, S.B. Golub, F.M. Rogerson, A.J. Fosang, ADAMTS-5 deficiency does not block aggrecanolysis at preferred cleavage sites in the chondroitin sulfate-rich region of aggrecan, J. Biol. Chem. 282 (2007) 8632-8640.

[54] P.J. Koshy, C.J. Lundy, A.D. Rowan, et al., The modulation of matrix metalloproteinase and ADAM gene expression in human chondrocytes by interleukin-1 and oncostatin M: a time-course study using real-time quantitative reverse transcription-polymerase chain reaction, Arthritis Rheum 46 (2002) 961-967.

[55] C.B. Little, C.T. Meeker, S.B. Golub, et al., Blocking aggrecanase cleavage in the aggrecan interglobular domain abrogates cartilage erosion and promotes cartilage repair, J. Clin. Invest 117 (2007) 1627-1636.

[56] H. Stanton, FM. Rogerson, C.J. East, et al., ADAMTS5 is the major aggrecanase in mouse cartilage in vivo and in vitro, Nature 434 (2005) 648-652.

[57] C. Gendron, M. Kashiwagi, N.H. Lim, et al., Proteolytic activities of human ADAMTS-5: comparative studies with ADAMTS-4, J. Biol. Chem. 282 (2007) 18294-18306.

[58] N. Al Fakhri, J. Wilhelm, M. Hahn, et al., Increased expression of dis-integrin-metalloproteinases ADAM-15 and ADAM-9 following upregu-lation of integrins alpha5betal and alphavbeta3 in atherosclerosis, J. Cell Biochem 89 (2003) 808-823.

[59] B. Cauwe, P.E. Steen, G. Opdenakker, The biochemical, biological, and pathological kaleidoscope of cell surface substrates processed by matrix metalloproteinases, Crit. Rev. Biochem. Mol. Biol. 42 (2007) 113-185.

[60] M.P. Sanderson, P.J. Dempsey, A.J. Dunbar, Control of ErbB signaling through metalloprotease mediated ectodomain shedding of EGFlike factors, Growth Factors 24 (2006) 121-136.

[61] K. Iba, R. Albrechtsen, B.J. Gilpin, F. Loechel, U.M. Wewer, Cysteine-rich domain of human ADAM 12 (meltrin alpha) supports tumor cell adhesion, Am. J. Pathol 154 (1999) 1489-1501.

[62] K. Iba, R. Albrechtsen, B. Gilpin, et al., The cysteine-rich domain of human ADAM 12 supports cell adhesion through syndecans and triggers signaling events that lead to betal integrin-dependent cell spreading, J. Cell Biol. 149 (2000) 1143-1156.

[63] C. Wild-Bode, K. Fellerer, J. Kugler, C. Haass, A. Capell, A basolateral sorting signal directs ADAM10 to adherens junctions and is required for its function in cell migration, J. Biol. Chem. 281 (2006) 23824-23829.

[64] C. Frohlich, R. Albrechtsen, L. Dyrskjot, L. Rudkjaer, T.F. Orntoft, U.M. Wewer, Molecular profiling of ADAM12 in human bladder cancer, Clin. Cancer Res. 12 (2006) 7359-7368.

[65] N. Ishikawa, Y. Daigo, W. Yasui, et al., ADAM8 as a novel serological and histochemical marker for lung cancer, Clin. Cancer Res. 10 (2004) 8363-8370

[66] S. Porter, S.D. Scott, E.M. Sassoon, et al., Dysregulated expression of adamalysin-thrombospondin genes in human breast carcinoma, Clin. Cancer Res. 10 (2004) 2429-2440.

[67] S.Y. Sung, H. Kubo, K. Shigemura, et al., Oxidative stress induces ADAM9 protein expression in human prostate cancer cells, Cancer Res. 66 (2006) 9519-9526. 
[68] D.J. Bevitt, Z. Li, J.L. Lindrop, M.D. Barker, M.P. Clarke, N. McKie, Analysis of full length ADAMTS6 transcript reveals alternative splicing and a role for the $5^{\prime}$ untranslated region in translational control, Gene 359 (2005) 99-110.

[69] D.P. Cerretti, R.F. DuBose, R.A. Black, N. Nelson, Isolation of two novel metalloproteinase-disintegrin (ADAM) cDNAs that show testis-specific gene expression, Biochem. Biophys. Res. Commun 263 (1999) 810-815.

[70] N. Hotoda, H. Koike, N. Sasagawa, S. Ishiura, A secreted form of human ADAM9 has an alpha-secretase activity for APP, Biochem. Biophys. Res. Commun 293 (2002) 800-805.

[71] L. Howard, R.A. Maciewicz, C.P. Blobel, Cloning and characterization of ADAM28: evidence for autocatalytic pro-domain removal and for cell surface localization of mature ADAM28, Biochem. J. 348 (Pt 1) (2000) 21-27.

[72] T. Katagiri, Y. Harada, M. Emi, Y. Nakamura, Human metalloprotease/ disintegrin-like (MDC) gene: exon-intron organization and alternative splicing, Cytogenet. Cell Genet. 68 (1995) 39-44.

[73] T. Kurisaki, S. Wakatsuki, A. Sehara-Fujisawa, Meltrin beta mini, a new ADAM19 isoform lacking metalloprotease and disintegrin domains, induces morphological changes in neuronal cells, FEBS Lett. 532 (2002) 419-422.

[74] R.M. Ortiz, I. Karkkainen, A.P. Huovila, Aberrant alternative exon use and increased copy number of human metalloproteasedisintegrin ADAM15 gene in breast cancer cells, Genes Chromosomes Cancer 41 (2004) 366-378.

[75] K. Sagane, Y. Ohya, Y. Hasegawa, I. Tanaka, Metalloproteinase-like, disintegrin-like, cysteine-rich proteins MDC2 and MDC3: novel human cellular disintegrins highly expressed in the brain, Biochem. J. 334 (Pt 1) (1998) 93-98.

[76] S.D. Wainwright, J. Bondeson, C.E. Hughes, An alternative spliced transcript of ADAMTS4 is present in human synovium from OA patients, Matrix Biol. 25 (2006) 317-320.

[77] R. Yavari, C. Adida, P. Bray-Ward, M. Brines, T. Xu, Human metallo-protease-disintegrin Kuzbanian regulates sympathoadrenal cell fate in development and neoplasia, Hum. Mol. Genet. 7 (1998) 1161-1167.

[78] Y.J. Liu, Y. Xu, Q. Yu, Full-length ADAMTS-1 and the ADAMTS-1 fragments display pro- and antimetastatic activity, respectively, Oncogene 25 (2006) 2452-2467.

[79] J.C. Rodriguez-Manzaneque, A.B. Milchanowski, E.K. Dufour, R. Leduc, M.L. Iruela-Arispe, Characterization of METH-1/ADAMTS1 processing reveals two distinct active forms, J. Biol. Chem. 275 (2000) 33471-33479.

[80] C.P. Blobel, ADAMs: key components in EGFR signalling and development, Nat. Rev. Mol. Cell Biol. 6 (2005) $32-43$.

[81] B. Schafer, B. Marg, A. Gschwind, A. Ullrich, Distinct ADAM metal-loproteinases regulate G protein-coupled receptor-induced cell proliferation and survival, J. Biol. Chem. 279 (2004) 47929-47938.

[82] A. Gschwind, S. Hart, O.M. Fischer, A. Ullrich, TACE cleavage of proamphiregulin regulates GPCR-induced proliferation and motility of cancer cells, EMBO J 22 (2003) 2411-2421.

[83] Q. Zhang, S.M. Thomas, V.W. Lui, et al., Phosphorylation of TNF-alpha converting enzyme by gastrin-releasing peptide induces amphiregulin release and EGF receptor activation, Proc. Natl. Acad. Sci. USA 103 (2006) 6901-6906.

[84] P.P. Ongusaha, J.C. Kwak, A.J. Zwible, et al., HB-EGF is a potent inducer of tumor growth and angiogenesis, Cancer Res. 64 (2004) $5283-5290$

[85] K. Horiuchi, S. Le Gall, M. Schulte, et al., Substrate selectivity of epidermal growth factor-receptor ligand sheddases and their regulation by phorbol esters and calcium influx, Mol. Biol. Cell 18 (2007) 176-188.

[86] U. Sahin, C.P Blobel, Ectodomain shedding of the EGF-receptor ligand epigen is mediated by ADAM17, FEBS Lett. 581 (2007) 41-44.

[87] B. Santiago-Josefat, C. Esselens, J.J. Bech-Serra, J. Arribas, Post-transcrip-tional up-regulation of ADAM17 upon epidermal growth factor receptor activation and in breast tumors, J. Biol. Chem. 282 (2007) 8325-8331.

[88] K. Ito, I. Okamoto, N. Araki, et al., Calcium influx triggers the sequential proteolysis of extracellular and cytoplasmic domains of Ecadherin, leading to loss of beta-catenin from cell-cell contacts, Oncogene 18 (1999) 7080-7090.

[89] T. Maretzky, K. Reiss, A. Ludwig, et al., ADAM1O mediates E-cadherin shedding and regulates epithelial cell-cell adhesion, migration, and beta-catenin translocation, Proc. Natl. Acad. Sci. USA 102 (2005) 9182-9187.

[90] M. Shtutman, J. Zhurinsky, I. Simcha, et al., The cyclin Dl gene is a target of the beta-catenin/LEF-1 pathway, Proc. Natl. Acad. Sci. USA 96 (1999) 5522-5527.

[91] M. Kveiborg, C. Frohlich, R. Albrechtsen, et al., A role for ADAM12 in breast tumor progression and stromal cell apoptosis, Cancer Res. 65 (2005) 4754-4761. 
Published in: Biochimie (2008), vol. 90, iss. 2, pp. 369-379

Status: Postprint (Author's version)

[92] J.S. Fridman, E. Caulder, M. Hansbury, et al., Selective inhibition of ADAM metalloproteases as a novel approach for modulating ErbB pathways in cancer, Clin. Cancer Res. 13 (2007) 1892-1902.

[93] K. Bajou, C. Maillard, M. Jost, et al., Host-derived plasminogen activator inhibitor-1 (PAI-1) concentration is critical for in vivo tumoral angiogenesis and growth, Oncogene 23 (2004) 6986-6990.

[94] I.J. Fidler, L.M. Ellis, The implications of angiogenesis for the biology and therapy of cancer metastasis, Cell 79 (1994) 185-188.

[95] D. Hanahan, J. Folkman, Patterns and emerging mechanisms of the angiogenic switch during tumorigenesis, Cell 86 (1996) $353-364$.

[96] M. Jost, A.R. Folgueras, F. Frerart, et al., Earlier onset of tumoral angiogenesis in matrix metalloproteinase-19-deficient mice, Cancer Res. 66 (2006) 5234-5241.

[97] A. Noel, C. Maillard, N. Rocks, et al., Membrane associated proteases and their inhibitors in tumour angiogenesis, J. Clin. Pathol 57 (2004) 577-584.

[98] R. Roy, B. Zhang, M.A. Moses, Making the cut: protease-mediated regulation of angiogenesis, Exp. Cell Res. 312 (2006) 608-622.

[99] F. Vazquez, G. Hastings, M.A. Ortega, et al., METH-1, a human ortholog of ADAMTS-1, and METH-2 are members of a new family of proteins with angio-inhibitory activity, J. Biol. Chem. 274 (1999) 23349-23357.

[100] M.L. Iruela-Arispe, M. Lombardo, H.C. Krutzsch, J. Lawler, D.D. Roberts, Inhibition of angiogenesis by thrombospondin-1 is mediated by 2 independent regions within the type 1 repeats, Circulation 100 (1999) 1423-1431.

[101] J. Lawler, The functions of thrombospondin-1 and-2, Curr. Opin. Cell Biol. 12 (2000) 634-640.

[102] A. Luque, D.R. Carpizo, M.L. Iruela-Arispe, ADAMTS1/METH1 inhibits endothelial cell proliferation by direct binding and sequestration of VEGF165, J. Biol. Chem. 278 (2003) 23656-23665.

[103] K. Kuno, K. Bannai, M. Hakozaki, K. Matsushima, K. Hirose, The carboxyl-terminal half region of ADAMTS-1 suppresses both tumorige-nicity and experimental tumor metastatic potential, Biochem. Biophys. Res. Commun 319 (2004) 1327-1333.

[104] B. Herren, E.W. Raines, R. Ross, Expression of a disintegrin-like protein in cultured human vascular cells and in vivo, FASEB J 11 (1997) 173-180.

[105] V. Trochon-Joseph, D. Martel-Renoir, L.M. Mir, et al., Evidence of anti-angiogenic and antimetastatic activities of the recombinant disintegrin domain of metargidin, Cancer Res. 64 (2004) 2062-2069.

[106] K. Horiuchi, G. Weskamp, L. Lum, et al., Potential role for ADAM15 in pathological neovascularization in mice, Mol. Cell Biol. 23 (2003) 5614-5624.

[107] V. Beck, H. Herold, A. Benge, et al., ADAM15 decreases integrin alphavbeta3/vitronectin-mediated ovarian cancer cell adhesion and motility in an RGD-dependent fashion, Int. J. Biochem. Cell Biol. 37 (2005) 590-603.

[108] H. Lemjabbar, D. Li, M. Gallup, S. Sidhu, E. Drori, C. Basbaum, Tobacco smoke-induced lung cell proliferation mediated by tumor necrosis factor alpha-converting enzyme and amphiregulin, J. Biol. Chem. 278 (2003) 26202-26207.

[109] B.B. Zhou, M. Peyton, B. He, et al., Targeting ADAM-mediated ligand cleavage to inhibit HER3 and EGFR pathways in non-small cell lung cancer, Cancer Cell 10 (2006) 39-50.

[110] Y Shintani, S. Higashiyama, M. Ohta, et al., Overexpression of ADAM9 in non-small cell lung cancer correlates with brain metastasis, Cancer Res. 64 (2004) 4190-4196.

[111] N. Rocks, G. Paulissen, C.F. Quesada, et al., Expression of a disintegrin and metalloprotease (ADAM and ADAMTS) enzymes in human non-small-cell lung carcinomas (NSCLC), Br. J. Cancer 94 (2006) 724-730.

[112] S. Mochizuki, M. Shimoda, T. Shiomi, Y. Fujii, Y. Okada, ADAM28 is activated by MMP-7 (matrilysin-1) and cleaves insulin-like growth factor binding protein-3, Biochem. Biophys. Res. Commun 315 (2004) 79-84.

[113] T. Ohtsuka, T. Shiomi, M. Shimoda, et al., ADAM28 is overexpressed in human non-small cell lung carcinomas and correlates with cell proliferation and lymph node metastasis, Int. J. Cancer 118 (2006) 263-273.

[114] A. Schutz, W. Hartig, M. Wobus, J. Grosche, C. Wittekind, G. Aust, Expression of ADAM15 in lung carcinomas, Virchows Arch 446 (2005) 421-429.

[115] J.R. Dunn, D. Panutsopulos, M.W. Shaw, et al., METH-2 silencing and promoter hypermethylation in NSCLC, Br. J. Cancer 91 (2004) $1149-1154$. 
[116] T. Harada, A. Nishie, K. Torigoe, et al., The specific expression of three novel splice variant forms of human metalloprotease-like disintegrin-like cysteine-rich protein 2 gene in brain tissues and gliomas, Jpn. J. Cancer Res. 91 (2000) 1001-1006.

[117] G.M. D'Abaco, K. Ng, L. Paradiso, N.J. Godde, A. Kaye, U. Novak, ADAM22, expressed in normal brain but not in high-grade gliomas, inhibits cellular proliferation via the disintegrin domain, Neurosurgery 58 (2006) 179-186.

[118] M. Bohm, R. Gerlach, W.D. Beecken, T. Scheuer, I. Stier-Bruck, I. Scharrer, ADAMTS-13 activity in patients with brain and prostate tumors is mildly reduced, but not correlated to stage of malignancy and metastasis, Thromb. Res. 111 (2003) 33-37.

[119] J.R. Dunn, J.E. Reed, D.G. du Plessis, et al., Expression of ADAMTS-8, a secreted protease with antiangiogenic properties, is downregulated in brain tumours, Br. J. Cancer 94 (2006) 1186-1193.

[120] J. Held-Feindt, E.B. Paredes, U. Blomer, et al., Matrix-degrading proteases ADAMTS4 and ADAMTS5 (disintegrins and metalloproteinases with thrombospondin motifs 4 and 5) are expressed in human glioblastomas, Int. J. Cancer 118 (2006) 55-61.

[121] X. Zheng, F. Jiang, M. Katakowski, et al., Inhibition of ADAM17 reduces hypoxia-induced brain tumor cell invasiveness, Cancer Sci. 98 (2007) 674-684

[122] D. Wildeboer, S. Naus, Q.X. Amy Sang, J.W. Bartsch, A. Pagenstecher, Metalloproteinase disintegrins ADAM8 and ADAM19 are highly regulated in human primary brain tumors and their expression levels and activities are associated with invasiveness, J. Neuropathol. Exp. Neurol 65 (2006) 516-527.

[123] T. Kodama, E. Ikeda, A. Okada, et al., ADAM12 is selectively overex-pressed in human glioblastomas and is associated with glioblastoma cell proliferation and shedding of heparin-binding epidermal growth factor, Am. J. Pathol 165 (2004) $1743-1753$.

[124] J. Bertram, J.W. Peacock, L. Fazli, et al., Loss of PTEN is associated with progression to androgen independence, Prostate 66 (2006) 895-902

[125] T. Ichikawa, H. Suzuki, T. Ueda, A. Komiya, T. Imamoto, S. Kojima, Hormone treatment for prostate cancer: current issues and future directions, Cancer Chemother. Pharmacol 56 (Suppl. 1) (2005) 58-63.

[126] D.R. McCulloch, M. Harvey, A.C. Herington, The expression of the ADAMs proteases in prostate cancer cell lines and their regulation by dihydrotestosterone, Mol. Cell Endocrinol 167 (2000) 11-21.

[127] K. Shigemura, S.Y. Sung, H. Kubo, et al., Reactive oxygen species mediate androgen receptor- and serum starvation-elicited downstream signaling of ADAM9 expression in human prostate cancer cells, Prostate 67 (2007) 722-731.

[128] F.R. Fritzsche, M. Jung, C. Xu, et al., ADAM8 expression in prostate cancer is associated with parameters of unfavorable prognosis, Virch-ows Arch. 449 (2006) 628-636.

[129] B.H. Koo, D. Oh, S.Y. Chung, et al., Deficiency of von Willebrand factor-cleaving protease activity in the plasma of malignant patients, Thromb. Res. 105 (2002) 471-476.

[130] L. Oleksowicz, N. Bhagwati, M. DeLeon-Fernandez, Deficient activity of von Willebrand's factor-cleaving protease in patients with disseminated malignancies, Cancer Res. 59 (1999) 2244-2250.

[131] N.A. Cross, S. Chandrasekharan, N. Jokonya, et al., The expression and regulation of ADAMTS-1, -4, -5, -9, and -15, and TIMP-3 by TGFbetal in prostate cells: relevance to the accumulation of versican, Prostate 63 (2005) 269-275.

[132] R. Kuefer, K.C. Day, C.G. Kleer, et al., ADAM15 disintegrin is associated with aggressive prostate and breast cancer disease, Neoplasia 8 (2006) 319-329.

[133] M.L. Moss, J.M. White, M.H. Lambert, R.C. Andrews, TACE and other ADAM proteases as targets for drug discovery, Drug Discov. Today 6 (2001) 417-426.

[134] D.R. McCulloch, P. Akl, H. Samaratunga, A.C. Herington, D.M. Odorico, Expression of the disintegrin metalloprotease, ADAM-10, in prostate cancer and its regulation by dihydrotestosterone, insulin-like growth factor I, and epidermal growth factor in the prostate cancer cell model LNCaP, Clin. Cancer Res. 10 (2004) 314-323.

[135] N. Theret, O. Musso, B. Turlin, et al., Increased extracellular matrix remodeling is associated with tumor progression in human hepatocellular carcinomas, Hepatology 34 (2001) 82-88.

[136] H. Le Pabic, D. Bonnier, U.M. Wewer, et al., ADAM12 in human liver cancers: TGF-beta-regulated expression in stellate cells is associated with matrix remodeling, Hepatology 37 (2003) 1056-1066.

[137] M. Matsuo, H. Sakurai, Y. Ueno, O. Ohtani, I. Saiki, Activation of MEK/ERK and PI3K/Akt pathways by fibronectin requires integrin alphav-mediated ADAM activity in hepatocellular carcinoma: a novel functional target for gefitinib, Cancer Sci. 97 (2006) $155-162$. 
[138] M.M. Murillo, G. del Castillo, A. Sanchez, M. Fernandez, I. Fabregat, Involvement of EGF receptor and c-Src in the survival signals induced by TGF-betal in hepatocytes, Oncogene 24 (2005) 4580-4587.

[139] H. Le Pabic, A. L'Helgoualc'h, A. Coutant, et al., Involvement of the serine/threonine p70S6 kinase in TGF-betal -induced ADAM12 expression in cultured human hepatic stellate cells, J. Hepatol 43 (2005) 1038-1044.

[140] A. Atfi, E. Dumont, F. Colland, et al., The disintegrin and metalloproteinase ADAM12 contributes to TGF-\{beta $\}$ signaling through interaction with the type II receptor, J. Cell Biol. 178 (2007) 201-208.

[141] A. Mazzocca, R. Coppari, R. De Franco, et al., A secreted form of ADAM9 promotes carcinoma invasion through tumor-stromal interactions, Cancer Res. 65 (2005) 4728-4738

[142] M. Borrell-Pages, F. Rojo, J. Albanell, J. Baselga, J. Arribas, TACE is required for the activation of the EGFR by TGF-alpha in tumors, EMBO J 22 (2003) 1114-1124.

[143] R. Roy, U.M. Wewer, D. Zurakowski, S.E. Pories, M.A. Moses, ADAM 12 cleaves extracellular matrix proteins and correlates with cancer status and stage, J. Biol. Chem. 279 (2004) 51323-51330.

[144] P.M. McGowan, B.M. Ryan, A.D. Hill, E. McDermott, N. O'Higgins, M.J. Duffy, ADAM-17 expression in breast cancer correlates with variables of tumor progression, Clin. Cancer Res. 13 (2007) 2335-2343.

[145] P.A. Kenny, M.J. Bissell, Targeting TACE-dependent EGFR ligand shedding in breast cancer, J. Clin. Invest 117 (2007) $337-345$.

[146] Y. Mitsui, S. Mochizuki, T. Kodama, et al., ADAM28 is overexpressed in human breast carcinomas: implications for carcinoma cell proliferation through cleavage of insulin-like growth factor binding protein-3, Cancer Res. 66 (2006) 9913-9920.

[147] C. O'Shea, N. McKie, Y. Buggy, et al., Expression of ADAM-9 mRNA and protein in human breast cancer, Int. J. Cancer 105 (2003) 754-761

[148] A.M. Sieuwerts, M.E. Meijer-van Gelder, M. Timmermans, et al., How ADAM-9 and ADAM-11 differentially from estrogen receptor predict response to tamoxifen treatment in patients with recurrent breast cancer: a retrospective study, Clin. Cancer Res. 11 (2005) 7311 7321.

[149] S. Porter, P.N. Span, F.C. Sweep, et al., ADAMTS8 and ADAMTS15 expression predicts survival in human breast carcinoma, Int. J. Cancer 118 (2006) 1241-1247.

[150] S. Carl-McGrath, U. Lendeckel, M. Ebert, A. Roessner, C. Rocken, The disintegrin-metalloproteinases ADAM9, ADAM12, and ADAM15 are upregulated in gastric cancer, Int. J. Oncol 26 (2005) 17-24.

[151] T. Yoshimura, T. Tomita, M.F. Dixon, A.T. Axon, P.A. Robinson, J.E. Crabtree, ADAMs (a disintegrin and metalloproteinase) messenger RNA expression in Helicobacter pylori-infected, normal, and neoplastic gastric mucosa, J. Infect. Dis 185 (2002) 332-340.

[152] J.M. Cox, C.L. Clayton, T. Tomita, D.M. Wallace, P.A. Robinson, J.E. Crabtree, cDNA array analysis of cag pathogenicity islandassociated Helicobacter pylori epithelial cell response genes, Infect. Immun 69 (2001) 6970-6980.

[153] T. Joh, H. Kataoka, S. Tanida, et al., Helicobacter pylori-stimulated inter-leukin-8 (IL-8) promotes cell proliferation through transactivation of epidermal growth factor receptor (EGFR) by disintegrin and metalloproteinase (ADAM) activation, Dig. Dis. Sci. 50 (2005) 2081-2089.

[154] F. Blanchot-Jossic, A. Jarry, D. Masson, et al., Up-regulated expression of ADAM17 in human colon carcinoma: co-expression with EGFR in neoplastic and endothelial cells, J. Pathol 207 (2005) 156-163.

[155] T. Hirao, D. Nanba, M. Tanaka, et al., Overexpression of ADAM9 enhances growth factor-mediated recycling of E-cadherin in human colon cancer cell line HT29 cells, Exp. Cell Res. 312 (2006) 331-339.

[156] G.E. Lind, K. Kleivi, G.I. Meling, et al., ADAMTS1, CRABP1, and NR3C1 identified as epigenetically deregulated genes in colorectal tumorigenesis, Cell Oncol 28 (2006) 259-272.

[157] D. Yamada, K. Ohuchida, K. Mizumoto, et al., Increased expression of ADAM 9 and ADAM 15 mRNA in pancreatic cancer, Anticancer Res. 27 (2007) 793-799.

[158] T. Masui, R. Hosotani, S. Tsuji, et al., Expression of METH-1 and METH-2 in pancreatic cancer, Clin. Cancer Res. 7 (2001) $3437-$ 3443.

[159] J. Ringel, R. Jesnowski, N. Moniaux, et al., Aberrant expression of a disintegrin and metalloproteinase 17/tumor necrosis factor-alpha converting enzyme increases the malignant potential in human pancreatic ductal adenocarcinoma, Cancer Res. 66 (2006) 9045-9053. 